Article

\title{
Performance Analysis of a User Selection Protocol in Cooperative Networks with Power Splitting Protocol-Based Energy Harvesting Over Nakagami-m/Rayleigh Channels
}

\author{
Tan N. Nguyen ${ }^{1}\left(\mathbb{D}\right.$, Minh Tran ${ }^{2, *} \mathbb{C}$, Thanh-Long Nguyen ${ }^{3}$, Duy-Hung $\mathrm{Ha}^{1}$ \\ and Miroslav Voznak ${ }^{1}$ (D) \\ 1 Faculty of Electrical Engineering and Computer Science, VSB-Technical University of Ostrava 17, \\ listopadu 15/2172, 70833 Ostrava-Poruba, Czech Republic; tan.nhat.nguyen.st@vsb.cz (T.N.N.); \\ duy.hung.ha.st@vsb.cz (D.-H.H.); miroslav.voznak@vsb.cz (M.V.) \\ 2 Optoelectronics Research Group, Faculty of Electrical and Electronics Engineering, \\ Ton Duc Thang University, Ho Chi Minh City 70000, Vietnam \\ 3 Center for Information Technology, Ho Chi Minh City University of Food Industry, \\ Ho Chi Minh City 70000, Vietnam; longngth@gmail.com \\ * Correspondence: tranhoangquangminh@tdtu.edu.vn; Tel.: +84-28-377-55-028
}

Received: 27 February 2019; Accepted: 15 April 2019; Published: 19 April 2019

\begin{abstract}
In this work, the system performance analysis of cooperative networks with power splitting protocol-based energy harvesting (EH) over Nakagami-m/Rayleigh channels is proposed. The exact-form expressions of the outage probability (OP) and ergodic capacity (EC) is demonstrated and derived. Using the proposed probabilistic models for wireless channels, we derive OP and EC as a research result. Finally, we conduct Monte Carlo simulations to verify a system performance analysis of the proposed system. The research results demonstrate the effectiveness of EH in the network over Nakagami-m/Rayleigh channels.
\end{abstract}

Keywords: amplify-and-forward (AF); outage probability; ergodic capacity; energy harvesting (EH); user selection

\section{Introduction}

Internet of Things (IoT) is considered a critical research area worldwide and has a significant impact on all activities in daily lives and industry [1-3]. However, because of numerous disadvantages, some industrial and civil aspects of IoT are difficult to achieve. Energy limitations are the most significant problem in the long-term operation of wireless networks. Prolonging lifetimes and battery recharging/replacement are not practical solution for wireless networks. Wireless energy harvesting $(\mathrm{EH})$ using radio frequency $(\mathrm{RF})$ is considered a promising solution for prolonging the operating time of devices in wireless, energy-constrained cooperative networks [4-6]. RF signals in EH wireless networks can transfer energy and information simultaneously. Cooperative networks are considered in connection with power sharing by helping the relay $(\mathrm{R})$ between the source $(\mathrm{S})$ and the destination (D) [6]. In EH cooperative networks, an energy-constrained R, by simultaneously harvesting energy and transferring information, helps maintain constant operation and connection without the use of external energy sources. The authors in [7] considered a dual-hop cognitive, inter-vehicular, relay-assisted communication system where all communication links are not line of sight, and their fading is modeled by the binary Rayleigh fading distribution. An analytic performance evaluation of the bit error rate (BER) of underlay decode-and-forward cognitive networks with the best relay selection over 
Rayleigh multipath fading channels was introduced in [8]. The authors in $[9,10]$ presented a general framework for modeling and evaluating the performance of dual-hop, decode-and-forward (DF) relaying schemes over independent and not necessarily identically distributed (INID) Nakagami- $m$ fading channels. The performance of dual-hop MIMO OSTBC transmission with multiple antennas employed at the transceiver and CSI-assisted AF relay over Nakagami-m fading is proposed in [11], and the performance of dual-hop, decode-and-forward relaying with relay selection (RS), analyzed over Nakagami fading channels, was studied in [12]. In our previous papers, we consider the EH over Rician fading channels. The titles of these papers are as follows: A Performance Analysis for Half-Duplex Bidirectional Sensor Networks under Hardware Impairments [12], Adaptive EH Relaying Protocol for a Two-Way Half Duplex System Network over Rician Fading Channels [13], Energy Harvesting-based Spectrum Access with Incremental Cooperation, Relay Selection, and Hardware Noises [14], and Performance Enhancement for EH Based Two-way Relay Protocols in Wireless Ad-Hoc Networks with Partial and Full Relay Selection Methods [15].

The problem of a two-hop EH cooperative relaying network was studied in [16]. In [17], a harvest-then-cooperate (HTC) protocol with AP points for S and R EH was investigated. The authors in [18] maximized the end-to-end achievable information transmission rate by assuming perfect channel state information (CSI) of the cooperative network with time switching (TS) and power splitting (PS) protocols. The authors in [19] investigated two-way relay beamforming optimization to maximize the achievable sum rate of simultaneous wireless information and power transfer (SWIPT) system with a full-duplex (FD) multiple-input multiple-output (MIMO), amplify-and-forward (AF) relay. To extend [19], the authors in [20] studied the joint optimization of two-way relay beamforming. Furthermore, the authors in [21] investigated SWIPT mechanisms in a relaying network with finite block length (FBL) codes. Moreover, the authors in [22] derived the theoretical symbol error probability (SEP) of cooperative systems with the best relay selection for Nakagami-m fading channels, and the authors in [23] proposed a novel DSTBC scheme with embedded adaptive DAF/amplify-and-forward (AAF) elements for data retransmission in multihop PLC networks with existing opportunistic listening (OL) protocols. The authors in [24] proposed a simple adaptive relaying protocol (ARP) for general relay networks, and in [25] we proposed a full rate DQOSTBC matrix with embedded adaptive DAF/AAF elements for four single-antenna relays. In spite of this research, cooperative relaying networks still need to be studied. This is the main aim of this paper.

There is very little research focus on both user selection protocol and EH with different Nakagami-m/Rayleigh channels. For this purpose, we analyzed the performance of user selection protocol cooperative networks with PS protocol-based EH over Nakagami-m/Rayleigh channels. Our article provides the following contributions. (i) We derive closed-form expressions of Outage probability (OP) and ergodic capacity (EC), (ii) propose user selection for choosing the best-received destination, (iii) investigate and compare the OP and EC with maximum and non-maximum capacity, and (iv) verify all analytical expressions by Monte Carlo simulations. The structure of the rest of the paper is as follows. Section 2 illustrates the system model, and Section 3 investigates the system performance. Section 4 proposes and discusses the research results, and conclusions are proposed in Section 5.

\section{System Model}

We consider an EH relay network with one S, multiple Ds, and one R as shown in Figure 1 [13,14]. In this system model, S, R, and D are working in a half-duplex (HD) mode with one antenna. The channel gain between node $S$ and the relay $\mathrm{R}\left(h_{R D}\right)$ is a Nakagami-m fading channel, so between the relay $\mathrm{R}$ and the destinations, $\mathrm{D}_{\mathrm{i}}$ as $h_{R D i}$ is represented by Rayleigh fading channels. In this model, the direct link between $S$ and D nodes is too weak without the help of a relay. The $\mathrm{EH}$ and information transmission (IT) for this proposed model system is presented in Figure 2. In this model, the transmission length time $T$ is divided into two slots. In the time slot $T / 2, \mathrm{R}$ harvests energy in $\rho P_{s}$ and receives information in $(1-\rho) P_{S}$ from $S$. The remaining half-time slot $T / 2$ is used for information transferring from $R$ to $D$ as in $[15,16]$. 


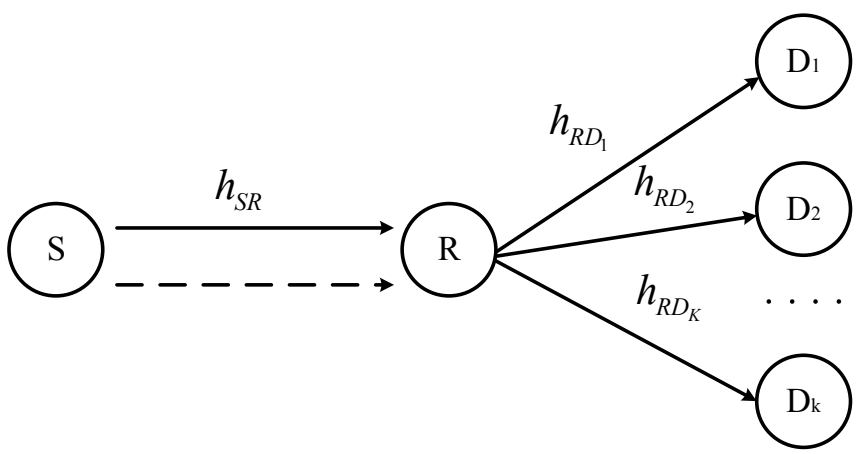

Figure 1. System model.



Figure 2. EH and IT of the proposed system.

In the first transmission phase, the received signal at $\mathrm{R}$ in period $T / 2$ time can be formulated by the following:

$$
y_{r}=\frac{1}{\sqrt{d_{s r}^{m}}} \sqrt{(1-\rho)} h_{s r} x_{s}+n_{r} .
$$

In this equation, $h_{s r}$ is $\mathrm{S}$ to $\mathrm{R}$ channel gain, $d_{s r}$ is the distance between $\mathrm{S}$ and $\mathrm{R}$, and $m$ denotes the path loss exponent. Here, $x_{s}$ is the transmitted signal at $\mathrm{S}, n_{r}$ is the additive white Gaussian noise (AWGN) with variance $\mathrm{N}_{0}$, and $0<\rho<1$ is the PS ratio at the relay R. Moreover, $\mathrm{E}\left\{\left|x_{s}\right|^{2}\right\}=P_{s}, \mathrm{E}\{\bullet\}$ is the expectation operator, and $P_{S}$ is the average transmit power at $\mathrm{S}$.

After the first-time slot $T / 2$, the harvested power at $R$ could be obtained by

$$
P_{r}=\frac{E_{h}}{d_{s r}^{m}(T / 2)}=\frac{\eta \rho P_{s}\left|h_{s r}\right|^{2}(T / 2)}{d_{s r}^{m}(T / 2)}=\frac{\eta \rho P_{s}\left|h_{s r}\right|^{2}}{d_{s r}^{m}}
$$

where $0<\eta \leq 1$ is the energy conversion efficiency.

In the second time slot $T / 2$, R retransmits the information from $S$ to $D_{i}$. The received signal at the $n^{\text {th }}$ destination at the second slot time can be expressed as

$$
y_{d_{i}}=\frac{1}{\sqrt{d_{i}^{m}}} h_{r d_{i}} x_{r}+n_{d_{i}}
$$

where $i \in(1,2, \ldots, K), h_{r d i}$ is the $\mathrm{R}$ to the $i^{\text {th }} \mathrm{D}$ channel gain, $d_{i}$ is the $\mathrm{R}$ to the $\mathrm{D}$ distance, $n_{d i}$ is the additive white Gaussian noise (AWGN) with variance $\mathrm{N}_{0}$, and $\mathrm{E}\left\{\left|x_{r}\right|^{2}\right\}=P_{r}$.

Here, the AF protocol is considered, and the amplifying factor can be given by

$$
\beta=\frac{x_{r}}{y_{r}}=\sqrt{\frac{P_{r}}{\frac{(1-\rho) P_{s}\left|h_{s r}\right|^{2}}{d_{s r}^{m}}+N_{0}} .}
$$


From Equations (1), (3) and (4), the received signal can be rewritten as

$$
\begin{aligned}
& y_{d_{i}}=\frac{1}{\sqrt{d_{r d_{n}}^{m}}} h_{r d_{i}} \beta y_{r}+n_{d_{i}}=\frac{1}{\sqrt{d_{r d_{i}}^{m}}} h_{r d_{i}} \beta\left[\frac{1}{\sqrt{d_{s r}^{m}}} \sqrt{(1-\rho)} h_{s r} x_{s}+n_{r}\right]+n_{d_{i}} \\
& =\underbrace{\frac{\sqrt{(1-\rho)} h_{s r} x_{s} h_{r d_{i}} \beta}{\sqrt{d_{i}^{m}} \sqrt{d_{s r}^{m}}}}_{\text {signal }}+\underbrace{\frac{1}{\sqrt{d_{i}^{m}}} h_{r d_{i}} \beta n_{r}+n_{d_{i}}}_{\text {noise }}
\end{aligned}
$$

Therefore, the overall signal to noise ratio (SNR) from $\mathrm{S}$ to $\mathrm{D}$ can be given by

$$
S N R=\frac{\mathrm{E}\left\{\mid \text { signal }\left.\right|^{2}\right\}}{\mathrm{E}\left\{\mid \text { noise }\left.\right|^{2}\right\}}=\frac{\frac{(1-\rho) P_{s}\left|h_{s r}\right|^{2}\left|h_{r d_{i}}\right|^{2} \beta^{2}}{d_{i}^{m} d_{s r}^{m}}}{\frac{\left|h_{r d_{i}}\right|^{2} \beta^{2} N_{0}}{d_{i}^{m}}+N_{0}}=\frac{(1-\rho) P_{s}\left|h_{s r}\right|^{2}\left|h_{r d_{i}}\right|^{2}}{\left|h_{r d_{i}}\right|^{2} d_{s r}^{m} N_{0}+\frac{N_{0} d_{i}^{m} d_{s r}^{m}}{\beta^{2}}}
$$

After doing some algebra, using the fact that $\mathrm{N}_{0}<<\mathrm{P}_{\mathrm{r}}$ and denote $\gamma_{1}=\left|h_{s r}\right|^{2}, \gamma_{i}=\left|h_{r d_{i}}\right|^{2}$, Equation (6) can be rewritten as

$$
S N R=\frac{(1-\rho) P_{s} P_{r}\left|h_{s r}\right|^{2}\left|h_{r d_{i}}\right|^{2}}{\left|h_{r d_{i}}\right|^{2} d_{s r}^{m} P_{r} N_{0}+(1-\rho) P_{s}\left|h_{s r}\right|^{2} N_{0} d_{i}^{m}} .
$$

Combined with Equation (2), we have the SNR:

$$
S N R=\frac{\eta \rho(1-\rho) \psi\left|h_{s r}\right|^{2}\left|h_{r d_{i}}\right|^{2}}{\eta \rho\left|h_{r d_{i}}\right|^{2} d_{s r}^{m}+(1-\rho) d_{i}^{m}}
$$

where $\psi=P_{s} / N_{0}$.

In our proposed model, we assume that the S-R link belongs to a Nakagami-m fading channel and the $\mathrm{R}-\mathrm{D}_{\mathrm{i}}$ link belongs to a Rayleigh fading channel. In the next section, we derive the analytical expression of OP and EC as in [26-31].

\section{The System Performance}

As in previous work $[12,13,28,30]$, the closed-form expressions of OP and EC of this system model are derived in this section. At first, we will determine the probability density function (PDF) and the cumulative density function (CDF) of a random variable (RV) $\left|h_{s r}\right|^{2},\left|h_{r d_{i}}\right|^{2}$. As shown in [29], the PDF of RV $\gamma_{1}$ can be calculated by

$$
f_{\left|h_{s r}\right|^{2}}(x)=\frac{x^{m_{\left|h_{s r}\right|^{2}}-1}}{\left(m_{\left|h_{s r}\right|^{2}}-1\right) !\left(\Omega_{\left|h_{s r}\right|^{2}}\right)^{m_{\left|h_{s r}\right|^{2}}}} \exp \left(-\frac{x}{\Omega_{\left|h_{s r}\right|^{2}}}\right) .
$$

From Equation (9), the CDF of RV $\left|h_{s r}\right|^{2}$ can be obtained with the help of Equation (8.353.4) in [32].

$$
F_{\left|h_{s r}\right|^{2}}(x)=1-\exp \left(-\frac{x}{\Omega_{\left|h_{s r}\right|^{2}}}\right) \sum_{t=0}^{m_{\left|h_{s r}\right|^{2}}-1} \frac{x^{t}}{t !\left(\Omega_{\left|h_{s r}\right|^{2}}\right)^{t}}
$$

where $\Omega_{\left|h_{s r}\right|^{2}}=\frac{\lambda_{s r}}{m_{\left|h_{s r}\right|^{2}}} ; m_{\left|h_{s r}\right|^{2}}$ is the Nakagami-m parameter and note that the case of $m_{\left|h_{s r}\right|^{2}}=1$ corresponds to Rayleigh fading; $\lambda_{s r}=\frac{\mathrm{E}\left\{\left|h_{s r}\right|^{2}\right\}}{d_{s r}^{m}}$ is the mean of RV $\left|h_{s r}\right|^{2}$. 
Furthermore, we have the PDF and CDF of RV $\left|h_{r d_{i}}\right|^{2}$ as shown in [28] as follows:

$$
\begin{aligned}
& f_{\left|h_{r d_{i}}\right|^{2}}(x)=\frac{1}{\lambda_{r d_{i}}} e^{-x / \lambda_{r d_{i}}} \\
& F_{\left|h_{r d_{i}}\right|^{2}}(x)=1-e^{-x / \lambda_{r d_{i}}}
\end{aligned}
$$

where $\lambda_{r d_{i}}=\frac{E\left\{\left|h_{r d_{i}}\right|^{2}\right\}}{d_{i}^{m}}$ is the mean of RV $\left|h_{r d_{i}}\right|^{2}$.

In this system model, the best selection user $U S_{q}$ is analyzed as follows:

$$
\left|h_{r d}\right|^{2}=\max _{i=1,2, \ldots, K}\left(\left|h_{r d_{i}}\right|^{2}\right) .
$$

According to [19], the CDF of $\left|h_{r d}\right|^{2}$ can be given by

$$
F_{\left|h_{r d}\right|^{2}}(y)=\sum_{p=0}^{K}(-1)^{p} C_{K}^{p} \times e^{-p y / \lambda_{r d}}
$$

where we denote $C_{K}^{p}=\frac{K !}{p !(K-p) !}$, and $\lambda_{r d}$ is the mean of $R V\left|h_{r d}\right|^{2}$.

Finally, the PDF of random variable can be determined by

$$
f_{\left|h_{r d}\right|^{2}}(y)=\frac{1}{\lambda_{r d}} \sum_{p=0}^{K-1}(-1)^{p} C_{K-1}^{p} K \times e^{-(p+1) y / \lambda_{r d}} .
$$

From Equation (19), we have

$$
S N R=\frac{\eta \rho(1-\rho) \psi\left|h_{s r}\right|^{2}\left|h_{r d}\right|^{2}}{\eta \rho\left|h_{r d}\right|^{2} d_{s r}^{m}+(1-\rho) d_{i}^{m}} .
$$

\subsection{The Outage Probability (OP)}

Theorem 1 (OP-Closed Form). The closed-form expression of the OP of the proposed model system can be formulated as

$$
\begin{aligned}
& P_{\text {out }}=1-2 \exp \left[-\frac{z d_{s r}^{m}}{\Omega_{\left|h_{s r}\right|^{2}}(1-\rho) \psi}\right]_{t=0}^{m_{\gamma_{1}}-1} \sum_{n=0}^{t} \sum_{p=0}^{K-1} \frac{(-1)^{p} K \times C_{K-1}^{p} z^{\frac{n+t+1}{2}}\left(\lambda_{r d}\right)^{\frac{n-t-1}{2}}}{(t-n) ! n !\left(\Omega_{|h s r|^{2}} \psi\right)^{\frac{n+t+1}{2}}(p+1)^{\frac{n-t+1}{2}}} \\
& \left(\frac{d_{i}^{m}}{\eta \rho}\right)^{\frac{t-n+1}{2}}\left[\frac{d_{s r}^{m}}{(1-\rho)}\right]^{n} \times K_{n-t+1}\left(2 \sqrt{\frac{z d_{i}^{m}(p+1)}{\Omega_{\left|h_{s r}\right|^{2}} \eta \rho \psi \lambda_{r d}}}\right)
\end{aligned}
$$

Proof of Theorem 1. See Appendix A.

\subsection{Maximize Capacity}

In this section, we will find the PS factor to maximize capacity. The overall capacity from source to destination can be given by

$$
C_{s, d}=\frac{1}{2} \log _{2}(1+S N R)=\frac{1}{2} \log _{2}\left[1+\frac{\eta \rho(1-\rho) \psi\left|h_{s r}\right|^{2}\left|h_{r d}\right|^{2}}{\eta \rho\left|h_{r d}\right|^{2} d_{s r}^{m}+(1-\rho) d_{i}^{m}}\right] .
$$


Therefore, to maximize capacity, from Equation (18), we can see that $\max \left(C_{s, d}\right) \Leftrightarrow \max (S N R)$.

From Equation (8), SNR is a concave function of $\rho$, which, maximizing the $S N R$, can be given as follows:

$$
\rho^{*}=\frac{1}{1+\left|h_{r d}\right| \sqrt{\frac{\eta d_{s r}^{m}}{d_{i}^{m}}}} .
$$

Theorem 2 (EC-Closed Form). The closed-form expression of the EC in the maximize mode of the system model can be expressed as

$$
C_{A F}=\frac{1}{\ln 2} \sum_{v=1}^{N_{p}} \omega_{v} \frac{1-F_{S N R_{\max }}\left(x_{v}\right)}{1+x_{v}} .
$$

Proof of Theorem 2. See Appendix B.

\section{Numerical Results and Discussion}

Like in previous studies [33-36], we conducted Monte Carlo simulations to verify analytical expressions of the OP and EC of the proposed system in the above section. In addition, we investigated the effect of the primary system parameter on the system performance in terms of OP and EC. All other simulation parameters are listed in Table 1.

Table 1. Simulation parameters.

\begin{tabular}{ccc}
\hline Symbol & Name & Values \\
\hline$\eta$ & Energy harvesting efficiency & 0.8 \\
\hline$\lambda_{s r}$ & Mean of $\left|h_{s r}\right|^{2}$ & 0.5 \\
\hline$\lambda_{r d}$ & Mean of $\left|h_{r d}\right|^{2}$ & 0.5 \\
\hline$m_{\gamma_{1}}$ & Nakagami m-factor & 3 \\
\hline $\mathrm{z}$ & SNR threshold & 1 \\
\hline $\mathrm{P}_{\mathrm{s}} / \mathrm{N}_{0}$ & Source power to noise ratio & $0-20 \mathrm{~dB}$ \\
\hline $\mathrm{R}$ & Source rate & $0.5 \mathrm{bit} / \mathrm{s} / \mathrm{Hz}$ \\
\hline $\mathrm{K}$ & Number of users & $1-6$ \\
\hline $\mathrm{m}$ & Pathloss exponent & 3 \\
\hline$d_{s r}=d_{i}$ & $\begin{array}{c}\text { the distance of S-R link and } \\
\text { R-D link, respectively }\end{array}$ & 0.85 \\
\hline
\end{tabular}

Figure 3a,b plots the influence of $\mathrm{P}_{\mathrm{s}} / \mathrm{N}_{0}$ on the OP and EC of the proposed system. In Figure 3 , the main parameters are as follows: $\mathrm{K}=2, \mathrm{R}=0.5$, and $\rho=0.2$ and 0.6 . In this figure, the OP of the case $\rho=0.2$ and 06 and the maximum capacity are also proposed for comparison. It is observed that the simulation values of the OP match the values from the mathematical analysis. In connection with the effect of $\rho$, the OP decreases, and EC increases as $\rho$ varies from 0.2 to 0.6 . When $\mathrm{P}_{\mathrm{s}} / \mathrm{N}_{0}$ increases from 0 to $20 \mathrm{~dB}$, the OP decreases and EC significantly increases. Furthermore, the higher the value of $\rho$ is, the faster the OP decreases and the EC increases. In addition, we can see that the OP and EC of the model system in the maximum capacity case are better in comparison with the other cases, with other values of $\rho$. This can be observed based on the mathematical analysis in Equations (17) and (20). 


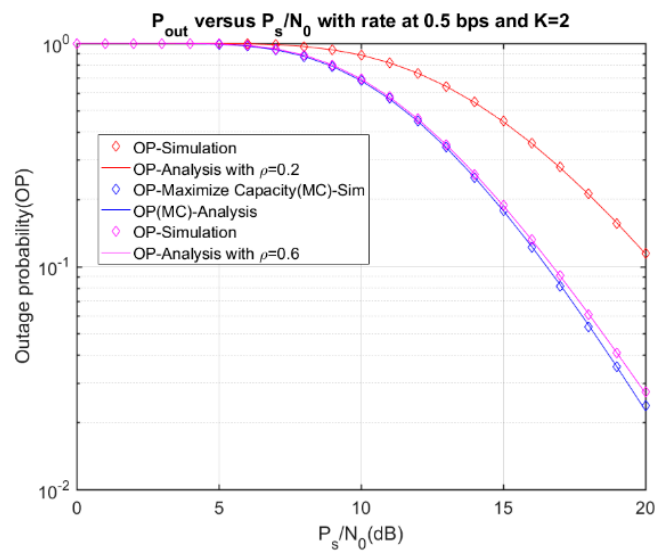

(a)

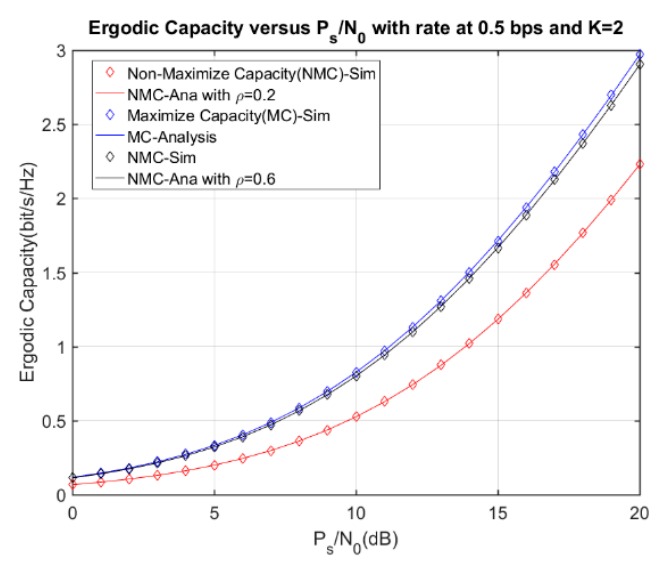

(b)

Figure 3. The outage probability (a) and ergodic capacity (b) versus $P_{S} / N_{0}$ while $K=2$.

Figure $4 \mathrm{a}, \mathrm{b}$ depicts the effect of $\mathrm{P}_{\mathrm{S}} / \mathrm{N}_{0}$ on the OP and the EC. We set $\mathrm{R}=0.5 \mathrm{bps}, \rho=0.5$, and $\mathrm{K}=1$, 3 , and 6 in Figure $4 \mathrm{a}$ and $\mathrm{R}=0.5 \mathrm{bps}, \rho=0.5$, and $K=1,3$, and 6 , respectively. From Figure $4 \mathrm{a}$, the OP decreases when $P_{S} / N_{0}$ increases from 0 to $20 \mathrm{~dB}$, and OP decreases faster with a higher $K$. On the other hand, the EC increases significantly, while $P_{s} / N_{0}$ rises from 0 to $20 \mathrm{~dB}$. Furthermore, the EC is higher with the higher $\mathrm{K}$ value. In all research results, the simulation and analytical results are the same.

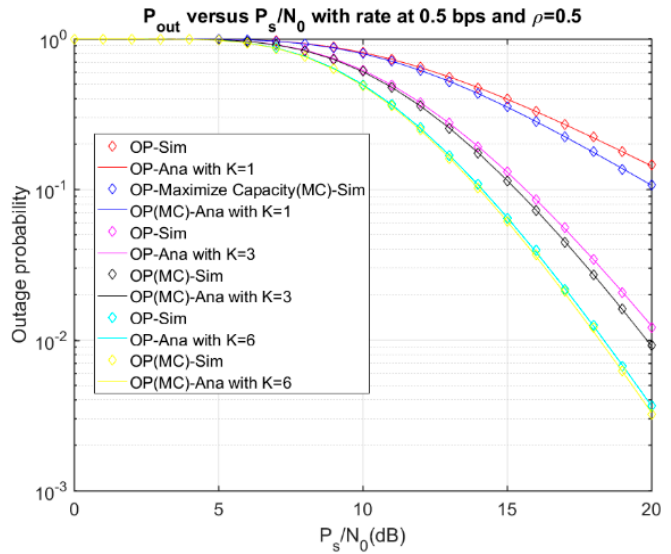

(a)

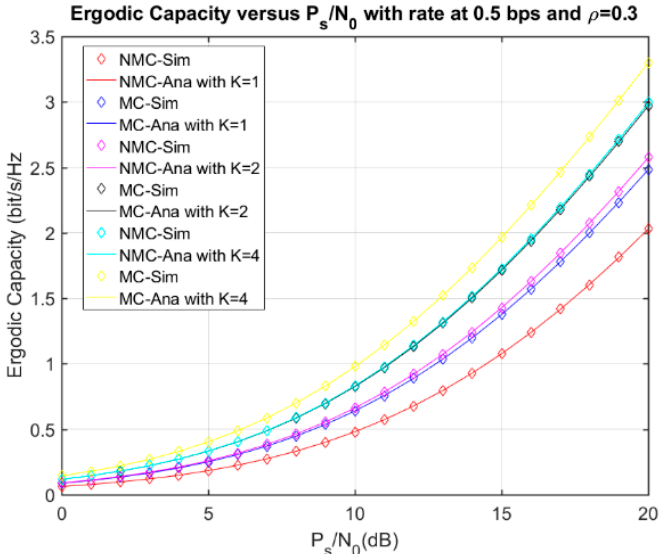

(b)

Figure 4. The outage probability (a) and ergodic capacity (b) versus $\mathrm{P}_{\mathrm{s}} / \mathrm{N}_{0}$ while $\rho=0.5$.

Moreover, Figure $5 \mathrm{a}, \mathrm{b}$ plots the influence of the ratio $\mathrm{P}_{\mathrm{S}} / \mathrm{N}_{0}$ on the OP and the EC of the model system. Here, the cases Ray-Ray, Naka-Ray in the non-maximize and maximize modes are compared with each other in the same system condition. In the simulation, we set the ratio $P_{\mathrm{S}} / \mathrm{N}_{0}$ increased from 0 to $20 \mathrm{~dB}, \rho=0.2$, and $\mathrm{K}=2$ for the OP and $\rho=0.4$ and $\mathrm{K}=3$ for the EC, respectively. Figure $5 \mathrm{a}$ shows that the OP decreases faster in the Naka-Ray case with maximum capacity compared with other cases. In the same way, the EC increases faster in the Ray-Ray case with non-maximum capacity in Figure $5 \mathrm{~b}$. Here we can see that the system performance in the maximum capacity case is better than in the non-maximum capacity case. Furthermore, the simulation results agreed with the mathematical analysis of the above section. 


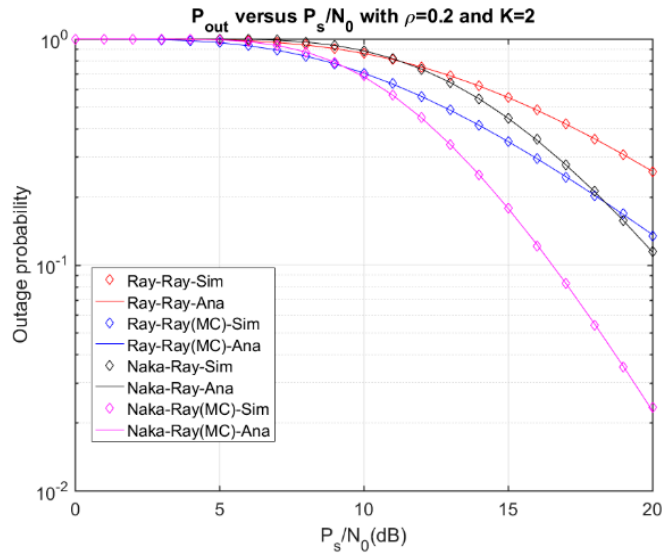

(a)

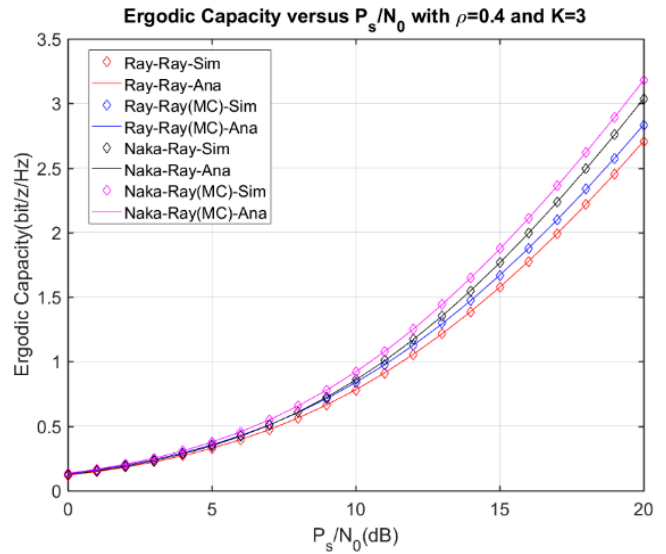

(b)

Figure 5. Comparison of the outage probability (a) and ergodic capacity (b) ersus $P_{\mathrm{S}} / \mathrm{N}_{0}$.

Figure 6 illustrates the OP versus the system factors $\eta$. In this case, we set $P_{\mathrm{S}} / \mathrm{N}_{0}=10 \mathrm{~dB}, \rho=0.3$, and $K=1$ and 4 in each case. From Figure 6, the OP decreases when $\eta$ increases from 0 to 1 , and the $\mathrm{OP}$ in the maximum capacity case is better than the non-maximum capacity case. Here, the simulation results agreed with the mathematical analysis of the above section. Furthermore, the OP and the EC of the proposed system in connection with the number of users are presented in Figure 7a,b. Similarly, the OP decreases and the EC increases, while the number of users varies from 0 to 10 and have better values in the maximum capacity case. In all of them, the simulation and analytical mathematical results agreed well with each other.

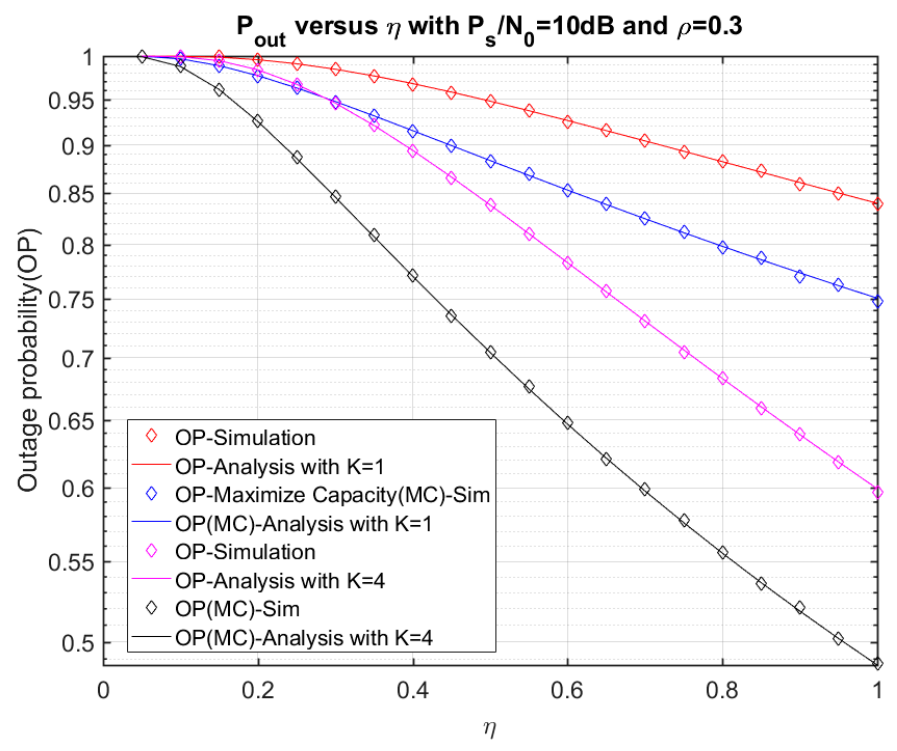

Figure 6. The outage probability versus $\eta$. 


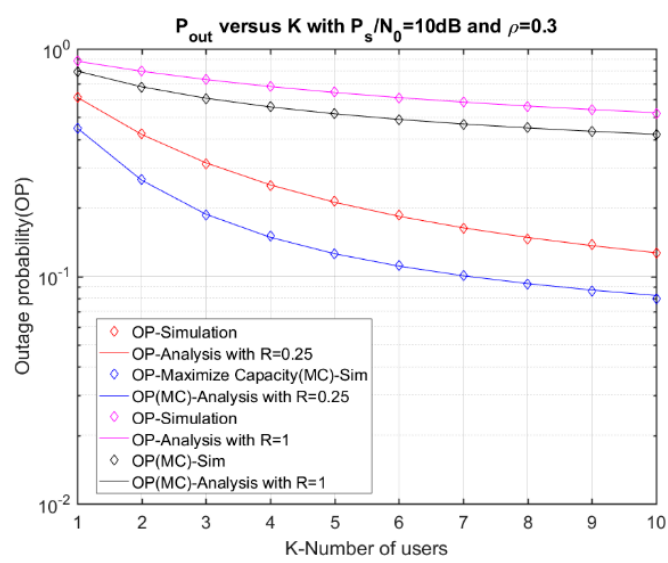

(a)

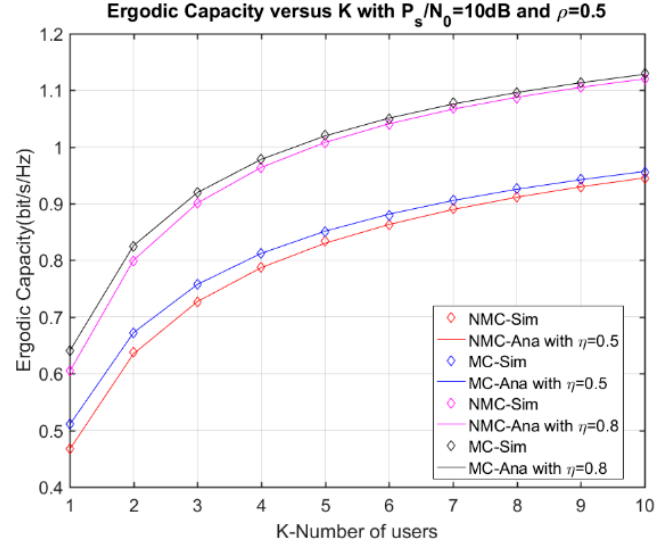

(b)

Figure 7. The outage probability (a) and ergodic capacity (b) versus the numbers of users.

\section{Conclusions}

This study proposes a performance analysis of a user selection protocol in cooperative networks with PS protocol-based EH over Nakagami-m/Rayleigh channels. The analytical closed-form expressions for the OP and the EC is derived. Moreover, the closed-form expression of the EC is derived in cases of maximum and non-maximum capacity. Finally, we use Monte Carlo simulations to derive analytical expressions in connection with all possible system parameters. From the research results, we can see that the simulation and the analytical analysis agree well.

Author Contributions: T.N.N. and M.T. created the main idea and developed the performance evaluation by analysis and simulation. M.T. wrote and revised the paper. T.-L.N. and D.-H.H. joined in the data analysis process. M.V. worked as an advisor to the group author of this project.

Funding: This research was funded by the Czech Ministry of Education, Youth and Sports within the grants No. SP2018/59 and SP2019/41 conducted at the VSB-Technical University of Ostrava.

Conflicts of Interest: The authors declare no conflicts of interest.

\section{Appendix A}

Outage probability can be calculated as

$$
P_{\text {out }}=\operatorname{Pr}(S N R<z)=\operatorname{Pr}\left(\frac{\eta \rho(1-\rho) \psi\left|h_{s r}\right|^{2}\left|h_{r d}\right|^{2}}{\eta \rho\left|h_{r d}\right|^{2} d_{s r}^{m}+(1-\rho) d_{i}^{m}}<z\right)
$$

where $z=2^{2 R}-1$ is a threshold, and $\mathrm{R}$ is the source rate.

Equation (A1) can be rewritten as

$$
\begin{aligned}
P_{\text {out }} & =\operatorname{Pr}\left\{\eta \rho(1-\rho) \psi\left|h_{s r}\right|^{2}\left|h_{r d}\right|^{2}<z \eta \rho\left|h_{r d}\right|^{2} d_{s r}^{m}+z(1-\rho) d_{i}^{m}\right\} \\
& =\operatorname{Pr}\left[\left|h_{s r}\right|^{2}<\frac{z \eta \rho\left|h_{r d}\right|^{2} d_{s r}^{m}+z(1-\rho) d_{i}^{m}}{\eta \rho(1-\rho) \psi\left|h_{r d}\right|^{2}}\right] \\
& =\operatorname{Pr}\left[\left|h_{s r}\right|^{2}<\frac{z d_{i}^{m}}{\eta \rho \psi\left|h_{r d}\right|^{2}}+\frac{z d_{s r}^{m}}{(1-\rho) \psi}\right] \\
P_{\text {out }}= & \int_{0}^{\infty} F_{\left|h_{s r}\right|^{2}}\left\{\frac{z d_{i}^{m}}{\eta \rho \psi\left|h_{r d}\right|^{2}}+\left.\frac{z d_{s r}^{m}}{(1-\rho) \psi}|| h_{r d}\right|^{2}\right\} f_{\left|h_{r d}\right|^{2}}\left(\left|h_{r d}\right|^{2}\right) d\left(\left|h_{r d}\right|^{2}\right) .
\end{aligned}
$$


From Equations (19) and (20), we have

$$
\begin{aligned}
& P_{\text {out }}=1-\int_{0}^{\infty} \exp \left[-\frac{\frac{z d_{i}^{m}}{\eta \rho \psi\left|h_{r d}\right|^{2}}+\frac{z d_{s r}^{m}}{(1-\rho) \psi}}{\Omega_{\left|h_{s r}\right|^{2}}}\right] \sum_{t=0}^{m_{\gamma_{1}}-1} \frac{\left[\frac{z d_{i}^{m}}{\eta \rho \psi\left|h_{r d}\right|^{2}}+\frac{z d_{s r}^{m}}{(1-\rho) \psi}\right]^{t}}{t !\left(\Omega_{\left|h_{s r}\right|^{2}}\right)^{t}} \\
& \times \frac{1}{\lambda_{q}} \sum_{p=0}^{K-1}(-1)^{p} C_{K-1}^{p} K \times e^{-(p+1)\left|h_{r d}\right|^{2} / \lambda_{q}} d\left(\left|h_{r d}\right|^{2}\right) \\
& P_{\text {out }}=1-\frac{\exp \left[-\frac{z d_{s r}^{m}}{\Omega_{\left|h_{s r}\right|^{2}}(1-\rho) \psi}\right]}{\lambda_{r d}} \int_{0}^{\infty} \sum_{t=0}^{\infty m_{\gamma_{1}}-1} \frac{\left.z \frac{z d_{i}^{m}}{\eta \rho \psi\left|h_{r d}\right|^{2}}+\frac{z d_{s r}^{m}}{(1-\rho) \psi}\right]^{t}}{t !\left(\Omega_{\left|h_{s r}\right|^{2}}\right)^{t}} \times \exp \left[-\frac{z d_{i}^{m}}{\Omega_{\left|h_{s r}\right|^{2}} \eta \rho \psi\left|h_{r d}\right|^{2}}\right] \\
& \times \sum_{p=0}^{K-1}(-1)^{p} C_{K-1}^{p} K \times e^{-(p+1)\left|h_{r d}\right|^{2} / \lambda_{r d} d\left(\left|h_{r d}\right|^{2}\right)}
\end{aligned}
$$

Now by applying the equation $(x+y)^{m}=\sum_{n=0}^{m}\left(\begin{array}{c}m \\ n\end{array}\right) x^{m-n} y^{n}$ to Equation (A5), the outage probability can demonstrate as follows:

$$
\begin{aligned}
& P_{\text {out }}=1-\frac{\exp \left[-\frac{z d_{s r}^{m}}{\Omega_{\left|h_{s r}\right|^{(1-\rho) \psi}}}\right.}{\lambda_{r d}} \\
& \int_{0}^{\infty} \sum_{t=0}^{m_{\gamma_{1}}-1} \sum_{n=0}^{t}\left(\begin{array}{c}
t \\
n
\end{array}\right) \frac{z^{t}}{t !\left(\Omega_{\left|h_{s r}\right|^{2}}\right)^{t}}\left(\frac{d_{i}^{m}}{\eta \rho \psi\left|h_{r d}\right|^{2}}\right)^{t-n}\left[\frac{d_{s r}^{m}}{(1-\rho) \psi}\right]^{n} \times \exp \left[-\frac{z d_{i}^{m}}{\Omega_{\left|h_{s r}\right|^{2}} \eta \rho \psi\left|h_{r d}\right|^{2}}\right] \\
& \times \sum_{p=0}^{K-1}(-1)^{p} C_{K-1}^{p} K \times e^{-(p+1)\left|h_{r d}\right|^{2} / \lambda_{r d} d\left(\left|h_{r d}\right|^{2}\right)} \\
& P_{\text {out }}=1-\frac{\exp \left[-\frac{z d_{s r}^{m}}{\Omega_{\left|h_{s r}\right|^{2}}(1-\rho) \psi}\right]}{\lambda_{r d}} \sum_{t=0}^{m_{\gamma_{1}}-1} \sum_{n=0}^{t} \sum_{p=0}^{K-1} \frac{(-1)^{p} K \times C_{K-1}^{p} z^{t}}{(t-n) ! n !\left(\Omega_{\left|h_{s r}\right|^{2}}\right)^{t} \psi^{t}}\left(\frac{d_{i}^{m}}{\eta \rho}\right)^{t-n}\left[\frac{d_{s r}^{m}}{(1-\rho)}\right]^{n} \\
& \times \int_{0}^{\infty}\left(\left|h_{r d}\right|^{2}\right)^{n-t} \times \exp \left[-\frac{z d_{i}^{m}}{\Omega_{\gamma_{1}} \eta \rho \psi\left|h_{r d}\right|^{2}}\right] \times e^{-(p+1)\left|h_{r d}\right|^{2} / \lambda_{r d} d\left(\left|h_{r d}\right|^{2}\right)}
\end{aligned}
$$

Using the Table of Integral Equation $(3.471,9)$ in [32], Equation (A7) can reformulated as

$$
\begin{aligned}
& P_{\text {out }}=1-2 \frac{\exp \left[-\frac{z d_{s r}^{m}}{\Omega_{\left|h_{s r}\right|^{2}}(1-\rho) \psi}\right]}{\lambda_{r d}} \sum_{t=0}^{m_{\gamma_{1}}-1} \sum_{n=0}^{t} \sum_{p=0}^{K-1} \frac{(-1)^{p} K \times C_{K-1}^{p} z^{t}}{(t-n) ! n !\left(\Omega_{\left|h_{s r}\right|^{2}}\right)^{t} \psi^{t}}\left(\frac{d_{i}^{m}}{\eta \rho}\right)^{t-n} \\
& {\left[\frac{d_{s r}^{m}}{(1-\rho)}\right]^{n}\left(\frac{z d_{i}^{m} \lambda_{r d}}{\Omega_{\left|h_{s r}\right|^{2}} \eta \rho \psi(p+1)}\right)^{\frac{n-t+1}{2}} \times K_{n-t+1}\left(2 \sqrt{\frac{z d_{i}^{m}(p+1)}{\Omega_{\left|h_{s r}\right|^{2}} \eta \rho \psi \lambda_{r d}}}\right)}
\end{aligned}
$$

where $K_{v}(\bullet)$ is the modified Bessel function of the second kind and the $\mathrm{v}$-th order.

\section{Appendix B}

It is easy to observe that $\frac{\partial^{2} S N R}{\partial \rho^{2}}$ is negative for $0<\rho<1$. Hence, we conclude that $S N R$ is a concave function of $\rho(0<\rho<1)$. We can find the value of $\rho$ that maximizes $S N R$ by differentiating the SNR concerning $\rho$, and we can then equate it to zero. After some algebraic calculations, we have the following possible solutions for $\rho^{*}$ :

$$
\rho^{*}=\frac{1}{1+\left|h_{r d}\right| \sqrt{\frac{\eta d_{s r}^{m}}{d_{i}^{m}}}} \text { or } \rho^{*}=\frac{1}{1-\left|h_{r d}\right| \sqrt{\frac{\eta d_{s r}^{m}}{d_{i}^{m}}}}
$$



the solution.

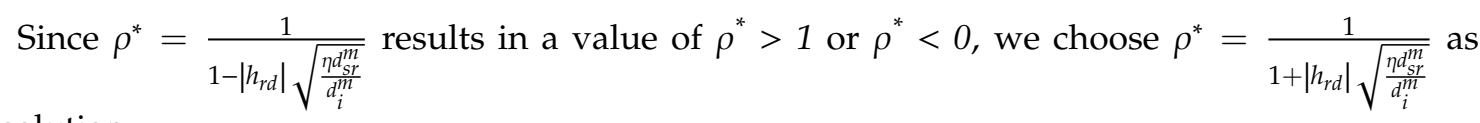

If Equation (A3) is placed into Equation (A1), $S N R_{\max }$ can be obtained as

$$
S N R_{\max }=\frac{\eta \psi\left|h_{s r}\right|^{2}\left|h_{r d}\right|^{2}}{\left(1+\left|h_{r d}\right| \sqrt{\frac{\eta d_{s r}^{m}}{d_{i}^{m}}}\right)^{2} d_{i}^{m}}
$$

EC analysis can be demonstrated as follows:

$$
\begin{gathered}
C_{A F}=\int_{0}^{\infty} f_{S N R_{\max }}(z) \log _{z}(1+z) d z=\frac{1}{\ln 2} \int_{0}^{\infty} \frac{1-F_{S N R_{\max }}(z)}{1+z} d z \\
F_{S N R_{\max }}(z)=\operatorname{Pr}\left(S N R_{\max }<z\right)=\operatorname{Pr}\left[\frac{\eta \psi\left|h_{s r}\right|^{2}\left|h_{r d}\right|^{2}}{\left(1+\left|h_{r d}\right| \sqrt{\left.\frac{\eta \eta_{s r}^{d m}}{d_{i}^{m}}\right)^{2}} d_{i}^{m}\right.}<z\right] \\
=\operatorname{Pr}\left[\left|h_{s r}\right|^{2}<\frac{\left(1+\left|h_{r d}\right| \sqrt{\frac{\eta d_{s r}^{m}}{d_{i}^{m}}}\right)^{2} z d_{i}^{m}}{\eta \psi\left|h_{r d}\right|^{2}}\right] \\
F_{S N R_{\max }}(z)=\int_{0}^{\infty} F_{\left|h_{s r}\right|^{2}}\left[\left.\frac{\left(1+\left|h_{r d}\right| \sqrt{\frac{\eta d_{s r}^{m}}{d_{i}^{m}}}\right)^{2} z d_{i}^{m}}{\eta \psi\left|h_{r d}\right|^{2}}|| h_{r d}\right|^{2} \mid f_{\left|h_{r d}\right|^{2}}\left(\left|h_{r d}\right|^{2}\right) d\left(\left|h_{r d}\right|^{2}\right) .\right.
\end{gathered}
$$

As in the previous section, we have

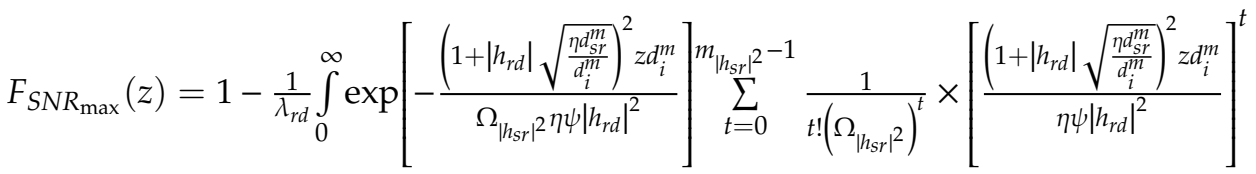

$$
\begin{aligned}
& \times \sum_{p=0}^{K-1}(-1)^{p} C_{K-1}^{p} K \times e^{-(p+1)\left|h_{r d}\right|^{2} / \lambda_{r d}} d\left(\left|h_{r d}\right|^{2}\right)
\end{aligned}
$$

In Equation (A14), we consider

$$
\begin{aligned}
& A=\exp \left[-\frac{\left(1+\left|h_{r d}\right| \sqrt{\frac{\eta d_{s t}^{m}}{d_{i}^{m}}}\right)^{2} z d_{i}^{m}}{\Omega_{\left|h_{s r}\right|^{2}} \eta \psi\left|h_{r d}\right|^{2}}\right]=\exp \left[-\frac{z d_{i}^{m}+2 z\left|h_{r d}\right| \sqrt{\eta d_{s r}^{m} d_{i}^{m}+z\left|h_{r d}\right|^{2} \eta d_{s r}^{m}}}{\Omega_{\left|h_{s r}\right|^{\prime}} \eta \psi\left|h_{r d}\right|^{2}}\right] \\
& =\exp \left[-\frac{z d_{i}^{m}}{\Omega_{\left|h_{s r}\right|^{2}} \eta \psi\left|h_{r d}\right|^{2}}\right] \exp \left[-\frac{2 z \sqrt{d_{s r}^{m} d_{i}^{m}}}{\Omega_{\left|h_{s r}\right|^{2}} \psi\left|h_{r d}\right| \sqrt{\eta}}\right] \exp \left[-\frac{z d_{s r}^{m}}{\Omega_{\left|h_{s r}\right|^{2}}}\right]
\end{aligned}
$$

We then apply a Taylor series as follows:

$$
\exp \left[-\frac{2 z \sqrt{d_{s r}^{m} d_{i}^{m}}}{\Omega_{\left|h_{s r}\right|^{2}} \psi\left|h_{r d}\right| \sqrt{\eta}}\right]=\sum_{q=0}^{\infty} \frac{\left(-\frac{2 z \sqrt{d_{s r}^{m} d_{i}^{m}}}{\Omega_{\left|h_{s r}\right|^{2}} \psi h_{r d} \mid \sqrt{\eta}}\right)^{q}}{q !}=\sum_{q=0}^{\infty} \frac{(-1)^{q}\left(\frac{2 z \sqrt{d_{s s}^{m} d_{i}^{m}}}{\Omega_{\left|h_{s r}\right|^{2} \sqrt{\eta}}}\right)^{q}}{q !}\left|h_{r d}\right|^{-q} .
$$


Equation (A15) can then be rewritten as

$$
A=\sum_{q=0}^{\infty} \frac{(-1)^{q}\left(\frac{2 z \sqrt{d_{s s}^{m} d_{i}^{m}}}{\Omega_{\left|h_{s r}\right|^{2}} \psi \sqrt{\eta}}\right)^{q}}{q !}\left|h_{r d}\right|^{-q} \times \exp \left[-\frac{z d_{s r}^{m}}{\Omega_{\left|h_{s r}\right|^{2}} \psi}\right] \exp \left[-\frac{z d_{i}^{m}}{\Omega_{\left|h_{s r}\right|^{2}} \eta \psi\left|h_{r d}\right|^{2}}\right] .
$$

If Equation (A14) is placed into Equation (A16) and the expression $(x+y)^{m}=\sum_{n=0}^{m}\left(\begin{array}{c}m \\ n\end{array}\right) x^{m-n} y^{n}$ is applied, we have

$$
\begin{aligned}
& F_{S N R_{\max }}(z)=1-\frac{1}{\lambda_{r d}} \int_{0}^{\infty} \sum_{q=0}^{\infty} \frac{(-1)^{q}\left(\frac{2 z \sqrt{d_{s s}^{m} r_{i}^{m m}}}{\Omega_{\mid h s r} 2^{q} \sqrt{\eta}}\right)^{q}}{q !}\left|h_{r d}\right|^{-q} \times \exp \left[-\frac{z d_{s r}^{m}}{\Omega_{\left|h_{s r}\right|^{2}}}\right] \exp \left[-\frac{z d_{i}^{m}}{\Omega_{\left|h_{s r}\right|^{2}} \eta \psi\left|h_{r d}\right|^{2}}\right]
\end{aligned}
$$

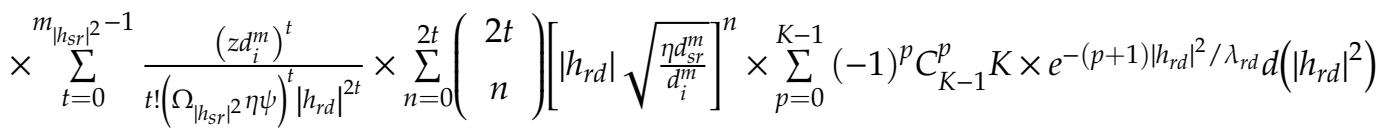

$$
\begin{aligned}
& F_{S N R_{\max }}(z)=1-\frac{1}{\lambda_{r d}} \sum_{t=0}^{m_{\left|h_{s r}\right|^{2}}-1} \sum_{n=0}^{2 t} \sum_{p=0}^{K-1} \sum_{q=0}^{\infty} \int_{0}^{\infty} \exp \left[-\frac{z d_{s r}^{m}}{\Omega_{\left|h_{s r}\right|^{2}}}\right] \exp \left[-\frac{z d_{i}^{m}}{\Omega_{\left|h_{s r \mid}\right|^{2}} \eta \psi\left|h_{r d}\right|^{2}}\right]
\end{aligned}
$$

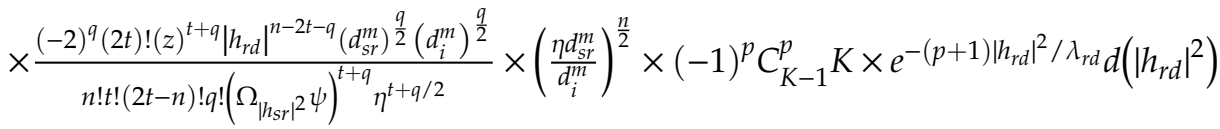

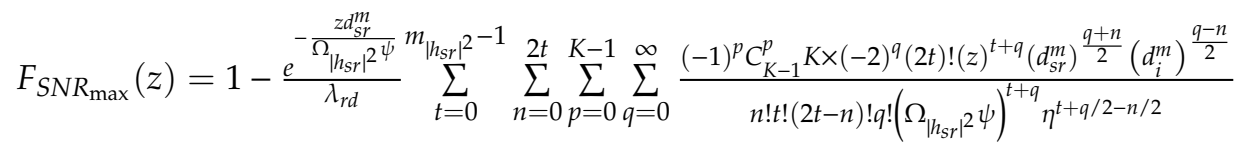

$$
\begin{aligned}
& \times \int_{0}^{\infty}\left|h_{r d}\right|^{n-2 t-q} \times \exp \left[-\frac{z d_{i}^{m}}{\Omega_{\left|h_{s r l}\right|^{2}} \eta \psi\left|h_{r d}\right|^{2}}\right] e^{-(p+1)\left|h_{r d}\right|^{2} / \lambda_{r d} d\left(\left|h_{r d}\right|^{2}\right)}
\end{aligned}
$$

Using the Table of Integral Equation $(3.471,9)$ in [32], Equation (A18) can be reformulated as

$$
\begin{aligned}
& F_{S N R_{\max }}(z)=1-\frac{2 e^{-\frac{z d_{s r}^{m}}{\Omega_{\mid h_{s r} 2^{2}}}}}{\lambda_{r d}} \sum_{t=0}^{m_{\left|h_{s r}\right|^{2}}} \sum_{n=0}^{2 t} \sum_{p=0}^{K-1} \sum_{q=0}^{\infty} \frac{(-1)^{p} C_{K-1}^{p} K \times(-2)^{q}(2 t) !(z)^{t+q}\left(d_{s r}^{m}\right)^{\frac{q+n}{2}}\left(d_{i}^{m}\right)^{\frac{q-n}{2}}}{n ! t !(2 t-n) ! q !\left(\Omega_{\left|h_{s r}\right|^{2}} \psi\right)^{t+q} \eta^{t+q / 2-n / 2}} \\
& \times\left[\frac{z d_{i}^{m} \lambda_{r d}}{(p+1) \Omega_{\left|h_{s r \mid}\right|^{2} \eta \psi}}\right]^{\frac{n-2 t-q+2}{4}} \times K_{\frac{n-2 t-q+2}{2}}\left[2 \sqrt{\frac{z d_{i}^{m}(p+1)}{\Omega_{\left|h_{s r \mid}\right|^{2} \eta \psi \lambda_{r d}}}}\right] \\
& F_{S N R_{\max }}(z)=1-2 e^{-\frac{z d_{s r}^{m}}{\Omega_{\left|h_{s r}\right|^{2}}}} \times \sum_{t=0}^{m_{\left|h_{s r \mid}\right|^{2}}} \sum_{n=0}^{2 t} \sum_{p=0}^{K-1} \sum_{q=0}^{\infty} \frac{(-1)^{p} C_{K-1}^{p} K \times(-2)^{q}(2 t) !\left(d_{s r}^{m}\right)^{\frac{q+n}{2}}\left(d_{i}^{m}\right)^{\frac{q-n-2 t+2}{4}}\left(\lambda_{r d}\right)^{\frac{n-2 t-q-2}{4}}}{n ! t !(2 t-n) ! q ! \eta !} \frac{\frac{2 t-n+q+2}{4}}{(p+1)^{\frac{n-2 t-q+2}{4}}} \\
& \times\left(\frac{z}{\Omega_{\left|h_{s r}\right|^{2}}}\right)^{\frac{n+2 t+3 q+2}{4}} \times K_{\frac{n-2 t-q+2}{2}}\left[2 \sqrt{\frac{z d_{i}^{m}(p+1)}{\Omega_{\left|h_{s \mid}\right|^{2} \psi} \eta \lambda_{r d}}}\right] \\
& C_{A F}=\frac{1}{\ln 2} \int_{0}^{\infty} \frac{1-F_{S N R_{\max }}(z)}{1+z} d z .
\end{aligned}
$$

We can observe that the involving integral in Equation (A22) is difficult to solve in a closed form. However, by changing the variable of the integration in Equation (B13) as $z=\tan \theta$, we have

$$
C_{A F}=\frac{1}{\ln 2} \int_{0}^{\infty} \frac{1-F_{S N R_{\max }}(z)}{1+z} d z=\frac{1}{\ln 2} \int_{0}^{\pi / 2} \frac{1-F_{S N R_{\max }}(\tan \theta)}{1+\tan \theta} \sec ^{2} \theta d \theta .
$$


We can then apply an efficient NP-point Gauss-Chebychev quadrature (GCQ) formula ([19] Equation (25.4.39)) from [20] to numerically derive the Proof of Theorem 2.

Where $x_{v}=\tan \left(\frac{\pi}{4} \cos \left[\frac{2 v-1}{2 N_{p}} \pi\right]+\frac{\pi}{4}\right)$, and $\omega_{v}=\frac{\pi^{2} \sin \left(\frac{2 v-1}{2 N_{p}} \pi\right)}{4 N_{p} \cos ^{2}\left(\frac{\pi}{4} \cos \left[\frac{2 v-1}{2 N_{p}} \pi\right]+\frac{\pi}{4}\right)}$.

\section{References}

1. Bi, S.; Ho, C.K.; Zhang, R. Wireless powered communication: Opportunities and challenges. IEEE Commun. Mag. 2015, 53, 117-125. [CrossRef]

2. Niyato, D.; Kim, D.I.; Maso, M.; Han, Z. Wireless Powered Communication Networks: Research Directions and Technological Approaches. IEEE Wirel. Commun. 2017, 2-11. [CrossRef]

3. Yu, H.; Lee, H.; Jeon, H. What is 5G? Emerging $5 \mathrm{G}$ Mobile Services and Network Requirements. Sustainability 2017, 9, 1848. [CrossRef]

4. Zhou, X.; Zhang, R.; Ho, C.K. Wireless Information and Power Transfer: Architecture Design and Rate-Energy Tradeoff. IEEE Trans. Commun. 2013, 61, 4754-4767. [CrossRef]

5. Lee, S.; Zhang, R.; Huang, K. Opportunistic Wireless Energy Harvesting in Cognitive Radio Networks. IEEE Trans. Commun. 2013, 12, 4788-4799. [CrossRef]

6. Chen, H.; Zhai, C.; Li, Y.; Vucetic, B. Cooperative Strategies for Wireless-Powered Communications: An Overview. IEEE Wirel. Commun. 2018, 25, 112-119. [CrossRef]

7. Duy, T.T.; Son, V.N.; Tung, V.T.; Alexandropoulos, G.C.; Duong, T.Q. Outage performance of cognitive cooperative networks with relay selection over double-Rayleigh fading channels. IET Commun. 2016, 10, 57-64. [CrossRef]

8. Ho-Van, K.; Sofotasios, P.C.; Alexandropoulos, G.C.; Freear, S. Bit error rate of underlay decode-and-forward cognitive networks with best relay selection. J. Commun. Netw. 2015, 17, 162-171. [CrossRef]

9. Alexandropoulos, G.C.; Papadogiannis, A.; Sofotasios, P.C. A Comparative Study of Relaying Schemes with Decode and Forward over Nakagami-Fading Channels. J. Comput. Netw. Commun. 2011, 2011, 1-14. [CrossRef]

10. Nguyen, T.; Tran, M.; Nguyen, T.-L.; Ha, D.-H.; Voznak, M. Multisource Power Splitting Energy Harvesting Relaying Network in Half-Duplex System over Block Rayleigh Fading Channel: System Performance Analysis. Electronics 2019, 8, 67. [CrossRef]

11. Yao, C.-H.; Pei, C.-X.; Guo, J. Performance analysis of two-way AF cooperative networks with relay selection over Nakagami-m fading channels. Inf. Technol. Appl. 2015, 9, 307-311.

12. Nguyen, T.; Quang Minh, T.; Tran, P.; Vozňák, M. Energy Harvesting over Rician Fading Channel: A Performance Analysis for Half-Duplex Bidirectional Sensor Networks under Hardware Impairments. Sensors 2018, 18, 1781. [CrossRef]

13. Nguyen, T.N.; Minh, T.H.; Tran, P.T.; Voznak, M. Adaptive Energy Harvesting Relaying Protocol for Two-Way Half Duplex System Network over Rician Fading Channel. Wirel. Commun. Mob. Comput. 2018, 2018, 1-10. [CrossRef]

14. Nguyen, T.N.; Duy, T.T.; Luu, G.T.; Tran, P.T.; Vozňák, M. Energy Harvesting-based Spectrum Access with Incremental Cooperation, Relay Selection and Hardware Noises. Radioengineering 2017, 26, 240-250. [CrossRef]

15. Nguyen, T.N.; Minh, T.H.; Tran, P.T.; Voznak, M.; Duy, T.T.; Nguyen, T.L.; Tin, P.T. Performance Enhancement for Energy Harvesting Based Two-way Relay Protocols in Wireless Ad-hoc Networks with Partial and Full Relay Selection Methods. Ad Hoc Netw. 2019, 84, 178-187. [CrossRef]

16. Gündüz, D.; Devillers, B. Two-hop Communication with Energy Harvesting. In Proceedings of the 2011 4th IEEE International Workshop on Computational Advances in Multi-Sensor Adaptive Processing (CAMSAP), San Juan, Puerto Rico, 13-16 December 2011. [CrossRef]

17. Chen, H.; Li, Y.; Rebelatto, J.L.; Uchoa-Filho, B.F.; Vucetic, B. Harvest-Then-Cooperate: Wireless-Powered Cooperative Communications. IEEE Trans. Signal Process. 2015, 63, 1700-1711. [CrossRef]

18. Xiong, K.; Fan, P.; Zhang, C.; Letaief, K.B. Wireless Information and Energy Transfer for Two-Hop Non-Regenerative MIMO-OFDM Relay Networks. IEEE J. Sel. Areas Commun. 2015, 33, 1595-1611. [CrossRef] 
19. Okandeji, A.A.; Khandaker, M.R.; Wong, K.K. Two-way Beamforming Optimization for Full-duplex SWIPT Systems. In Proceedings of the 2016 24th European Signal Processing Conference (EUSIPCO), Budapest, Hungary, 29 August-2 September 2016. [CrossRef]

20. Okandeji, A.A.; Khandaker, M.R.; Wong, K.K.; Zheng, Z. Joint Transmit Power and Relay Two-Way Beamforming Optimization for Energy-Harvesting Full-Duplex Communications. In Proceedings of the 2016 IEEE Globecom Workshops (GC Wkshps), Washington, DC, USA, 4-8 December 2016. [CrossRef]

21. Hu, Y.; Zhu, Y.; Schmeink, A. Simultaneous Wireless Information and Power Transfer in Relay Networks with Finite Blocklength Codes. In Proceedings of the 2017 23rd Asia-Pacific Conference on Communications (APCC), Perth, Australia, 11-13 December 2017. [CrossRef]

22. Halima, N.B.; Boujemaa, H. Exact and Approximate Symbol Error Probability of cooperative systems with best relay selection and all participating relaying using Amplify and Forward or Decode and Forward Relaying over Nakagami-m fading channels. KSII Trans. Internet Inf. Syst. 2018, 12, 81-108.

23. Tseng, S.-M.; Lee, T.-L.; Ho, Y.-C.; Tseng, D.-F. Distributed space-time block codes with embedded adaptive AAF/DAF elements and opportunistic listening for multihop power line communication networks. Int. J. Commun. Syst. 2015, 30, e2950. [CrossRef]

24. Li, Y.; Vucetic, B. On the Performance of a Simple Adaptive Relaying Protocol for Wireless Relay Networks. In Proceedings of the VTC Spring 2008-IEEE Vehicular Technology Conference, Singapore, 11-14 May 2008.

25. Tseng, S.-M.; Liao, C.-Y. Distributed Orthogonal and Quasi-Orthogonal Space-Time Block Code with Embedded AAF/DAF Matrix Elements in Wireless Relay Networks with Four Relays. Wirel. Pers. Commun. 2013, 75, 1187-1198. [CrossRef]

26. Katti, S.; Gollakota, S.; Katabi, D. Embracing Wireless Interference. ACM SIGCOMM Comput. Commun. Rev. 2007, 37, 397-408. [CrossRef]

27. Qin, J.; Zhu, Y.; Zhe, P. Broadband Analog Network Coding With Robust Processing for Two-Way Relay Networks. IEEE Commun. Lett. 2017, 21, 1115-1118. [CrossRef]

28. Nasir, A.A.; Zhou, X.; Durrani, S.; Kennedy, R.A. Relaying Protocols for Wireless Energy Harvesting and Information Processing. IEEE Trans. Commun. 2013, 12, 3622-3636. [CrossRef]

29. Deng, Y.; Wang, L.; Elkashlan, M.; Kim, K.J.; Duong, T.Q. Generalized Selection Combining for Cognitive Relay Networks Over Nakagami-m Fading. IEEE Trans. Signal Process. 2015, 63, 1993-2006. [CrossRef]

30. Nguyen, T.N.; Tran, P.T.; Minh, T.H.Q.; Voznak, M.; Sevcik, L. Two-Way Half Duplex Decode and Forward Relaying Network with Hardware Impairment over Rician Fading Channel: System Performance Analysis. Electron. Electr. Eng. 2018, 24, 74-78. [CrossRef]

31. Owen, D.B.; Abramowitz, M.; Stegun, I.A. Handbook of Mathematical Functions with Formulas, Graphs, and Mathematical Tables. Technometrics 1965, 7, 78. [CrossRef]

32. Gradshteyn, I.S.; Ryzhik, I.M. Table of Integrals, Series, and Products, 8th ed.; Daniel, Z., Victor, M., Eds.; Academic Press: Amsterdam, The Netherlands, 2015.

33. Bhatnagar, M.R. On the Capacity of Decode-and-Forward Relaying over Rician Fading Channels. IEEE Commun. Lett. 2013, 17, 1100-1103. [CrossRef]

34. Mouapi, A.; Hakem, N. A New Approach to Design Autonomous Wireless Sensor Node Based on RF Energy Harvesting System. Sensors 2018, 18, 133. [CrossRef]

35. Wang, C.; Li, J.; Yang, Y.; Ye, F. Combining Solar Energy Harvesting with Wireless Charging for Hybrid Wireless Sensor Networks. IEEE Trans. Mob. Comput. 2018, 17, 560-576. [CrossRef]

36. Chu, Z.; Zhou, F.; Zhu, Z.; Hu, R.Q.; Xiao, P. Wireless Powered Sensor Networks for Internet of Things: Maximum Throughput and Optimal Power Allocation. IEEE Internet Things J. 2018, 5, 310-321. [CrossRef]

(C) 2019 by the authors. Licensee MDPI, Basel, Switzerland. This article is an open access article distributed under the terms and conditions of the Creative Commons Attribution (CC BY) license (http://creativecommons.org/licenses/by/4.0/). 